
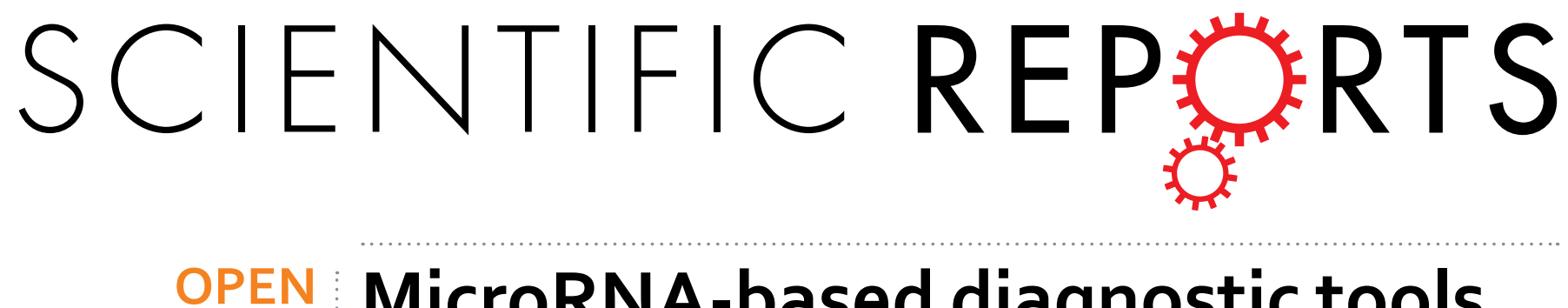

\title{
MicroRNA-based diagnostic tools for advanced fibrosis and cirrhosis in patients with chronic hepatitis
}

Received: 16 May 2016

Accepted: 20 September 2016

Published: 12 October 2016
$B$ and $C$

Kevin Appourchaux $x^{1,2,3}$, Safi Dokmak ${ }^{4}$, Matthieu Resche-Rigon ${ }^{5}$, Xavier Treton ${ }^{6}$, Martine Lapalus ${ }^{1,2,3}$, Charles-Henry Gattolliat ${ }^{1,2,3}$, Emmanuelle Porchet ${ }^{1,2,3}$, Michelle Martinot-Peignoux $x^{1,2,3}$, Nathalie Boyer ${ }^{1,2,3}$, Michel Vidaud ${ }^{7}$, Pierre Bedossa ${ }^{8}$, Patrick Marcellin ${ }^{1,2,3}$, Ivan Bièche ${ }^{7}$, Emilie Estrabaud ${ }^{1,2,3, *}$ \& Tarik Asselah ${ }^{1,2,3, *}$

Staging fibrosis is crucial for the prognosis and to determine the rapid need of treatment in patients with chronic hepatitis B (CHB) and C (CHC). The expression of 13 fibrosis-related microRNAs (miRNAs) (miR-20a, miR-21, miR-27a, miR-27b, miR-29a, miR-29c, miR-92a, miR-122, miR-146a, miR-155, miR-221, miR-222, and miR-224) was analyzed in 194 serums and 177 liver biopsies of patients with either $\mathrm{CHB}$ or $\mathrm{CHC}$ to develop models to diagnose advanced fibrosis and cirrhosis (Metavir F3-F4). In CHB patients, the model (serum miR-122, serum miR-222, platelet count and alkaline phosphatase) was more accurate than APRI and FIB-4 to discriminate in between mild and moderate fibrosis (F1-F2) and F3-F4 (AUC of CHB model: 0.85 vs APRI: 0.70 and FIB-4: 0.81 ). In CHC patients, the model (hepatic miR-122, hepatic miR-224, platelet count, albumin and alanine aminotransferase) was more accurate than both APRI and FIB-4 to discriminate in between patients with F3-F4 and F1-F2 (AUC of the CHC model $=0.93$ vs APRI: 0.86 and FIB-4: 0.79 ). Most of the miRNAs tested were differentially expressed in patients with $\mathrm{CHB}$ and $\mathrm{CHC}$. In particular, serum miR-122 was 28 -fold higher in patients with $\mathrm{CHB}$ than in those with CHC. Both CHB and CHC models may help for the diagnosis of advanced fibrosis and cirrhosis (F3-F4).

Chronic hepatitis $\mathrm{B}(\mathrm{CHB})$ and $\mathrm{C}(\mathrm{CHC})$ affect respectively 350 and 170 million individuals worldwide ${ }^{1}$. Patients with $\mathrm{CHB}$ and $\mathrm{CHC}$ are at high risk to develop liver fibrosis, cirrhosis and hepatocellular carcinoma (HCC) ${ }^{2}$. The evaluation of liver fibrosis is crucial for assessing the prognosis and the need of treatment ${ }^{3-6}$. Scoring systems (Knodell, Metavir, Ishak, etc.) provide a semi-quantitative assessment for individual clinical prognosis, cross-sectional, cohort studies and treatment trial. In patients with $\mathrm{CHB}$ and $\mathrm{CHC}$, the liver biopsy remains the gold standard to assess fibrosis despite inter-/intra-observer variations and sampling errors ${ }^{7}$.

Alternate non-invasive methods have been initially developed and evaluated in patients with $\mathrm{CHC}^{8}$ and increasingly evaluated in $\mathrm{CHB}^{9,10}$. They include transient elastography and serological scores such as the "aspartate aminotransferase (AST)-to-platelet ratio index" (APRI) ${ }^{11}$ and the "four factors-based fibrosis index"

${ }^{1}$ INSERM, UMR1149, Team «Physiopathologie et traitements des hépatites virales», Centre de Recherche sur I'Inflammation, and Université Denis Diderot Paris 7, site Bichat, BP 416, F-75018, Paris, France. ${ }^{2}$ Service d'hépatologie, PMAD Hôpital Beaujon, 100 Bd du Général Leclerc, Clichy la Garenne, 92110 Clichy Cedex, France. ${ }^{3}$ Laboratory of Excellence Labex INFLAMEX, PRES Paris Sorbonne Cité, Paris, France. ${ }^{4}$ Department of HPB Surgery and Liver Transplantation, Beaujon Hospital, Paris, France; Assistance Publique Hôpitaux de Paris, Paris, France; Université Paris VII Denis Diderot, Paris, France. ${ }^{5}$ Service de Biostatistique et Information Médicale, Hôpital SaintLouis, AP-HP, ECSTRA Team, Inserm UMR1153, Paris, France. ${ }^{6}$ Service de gastroentérologie, MICl et Assistance Nutritive, Hôpital Beaujon, Assistance Publique Hôpitaux de Paris, Clichy, France. ${ }^{7}$ UMR745 INSERM, Université Paris Descartes, Sorbonne Paris Cité, Faculté des Sciences Pharmaceutiques et Biologiques, Paris, France. ${ }^{8}$ Service d'anatomopathologie, Hôpital Beaujon, Assistance Publique Hôpitaux de Paris, Clichy, France. *These authors contributed equally to this work. Correspondence and requests for materials should be addressed to E.E. (email: emilie. estrabaud@inserm.fr) orT.A. (email: tarik.asselah@bjn.aphp.fr) 
$(\text { FIB-4 })^{12}$. Both index have a moderate sensitivity and accuracy to discriminate in between each of the stages of fibrosis of the Metavir score ${ }^{13,14}$. While the World Health Organization (WHO) recommends the use of APRI in resource-limited countries ${ }^{15,16}$, a recent study suggested that APRI and FIB-4 are not suitable enough for use in clinical practice in $\mathrm{CHB}$ patients ${ }^{10}$. Whereas serological scores are very suitable to detect the absence of fibrosis or cirrhosis and severe fibrosis, their performance remains low to diagnose moderate fibrosis (Metavir F2) ${ }^{8}$.

MicroRNAs (miRNAs) are small non-coding endogenous RNAs that regulate up to $60 \%$ of the expression of cellular mRNAs. Previous studies showed that several miRNAs regulate liver functions and are associated with $\mathrm{HCC}^{17}$. Moreover, different sets of miRNAs have been associated with $\mathrm{CHB}$ and $\mathrm{CHC}$-induced fibrosis and inflammatory liver diseases ${ }^{17,18}$. Nevertheless, those studies mainly focused on cirrhosis. Little is known about the level of miRNAs expression within intermediate stages of fibrosis.

MiR-122 enhances the replication of $\mathrm{HCV}^{19}$. We previously reported a reduction of the hepatic expression of miR-122 in patients with $\mathrm{CHC}$ at the most advanced stages of fibrosis ${ }^{20}$. MiR-122 has been suggested to interact with $\mathrm{HBV}$ genome and to inhibit its replication ${ }^{21}$. Interestingly, in patients with $\mathrm{CHB}$, the down-regulation of miR-122 may contribute to viral persistence and carcinogenesis ${ }^{22}$.

The main objective of the study was to identify miRNAs associated with fibrosis in order to establish miRNAs-based models to discriminate between patients with advanced fibrosis and cirrhosis (F3-F4) and those with mild to moderate fibrosis (F1-F2) in CHB and CHC. The expression profiles of 13 selected miRNAs were analyzed in serum and liver biopsies of patients with $\mathrm{CHB}$ and $\mathrm{CHC}$. Models to diagnose severe fibrosis and cirrhosis (F3-F4) in CHC and CHB were established based on multivariate analysis. Secondary objectives of this work were to study the correlation between the expression of miRNAs in serum and liver.

\section{Results}

Patients and baseline characteristics. In the total cohort ( $\mathrm{CHB}$ and $\mathrm{CHC}$ patients), 142 patients with mild (F1) to moderate (F2) fibrosis (F1-F2) and 138 patients with advanced fibrosis (F3) to cirrhosis (F4) (F3-F4) were included (Table S1). The mean age was 47 years in both patients with F1-F2 and those with F3- F4 (F3-F4). Patients with F1-F2 had lower body mass index (BMI) $(\mathrm{p}=0.034)$, alanine amino transferase (ALT) $(\mathrm{p}=0.0002)$, aspartate amino transferase (AST) $(p<0.0001)$, alkaline phosphatase (APL) $(p<0.0001)$, gamma glutamyltransferase (GGT) $(p<0.0001)$, total bilirubin $(p=0.005)$, glycemia $(p=0.0002)$ and cholesterol $(p<0.0001)$ than patients with F3-F4 (Table S1).

Patients with CHC were older $(\mathrm{p}<0.0001)$, had higher AST $(\mathrm{p}=0.025)$, ALP $(\mathrm{p}=0.0003)$, GGT $(\mathrm{p}<0.0001)$, glycemia $(\mathrm{p}=0.01)$, platelet count $(\mathrm{p}=0.04)$ and prothrombin time $(\mathrm{PT})(\mathrm{p}<0.0001)$ than patients with CHB (Table S2).

In the $\mathrm{CHB}$ cohort, 58 and 44 patients with respectively F1-F2 and F3-F4 were included. Thirty-nine out of the 103 patients were HBe antigen positive (data not shown). CHB patients with F1-F2 had lower AST $(p=0.047)$, $\operatorname{ALP}(p=0.0002)$, GGT $(p=0.001)$, glycemia $(p=0.027)$ and triglycerides $(p=0.035)$ than CHB patients with F3-F4. CHB patients with F1-F2 had higher platelet count $(\mathrm{p}=0.003), \mathrm{PT}(\mathrm{p}=0.011)$ and albumin $(\mathrm{p}=0.002)$ than CHB patients with F3-F4 (Table 1).

In the CHC cohort, 84 patients with F1-F2 and 94 with F3-F4 were enrolled (Table 1). The mean age at liver biopsy was 49 years. All genotypes were represented with $51.95 \%, 14.29 \%, 14.29 \%, 14.29 \%, 3.90 \%$ and $1.30 \%$ of patients with respectively genotypes $1,2,3,4,5$ and 6 . CHC patients with F1-F2 had lower ALT ( $\mathrm{p}=0.0002)$, AST $(p<0.0001)$, ALP $(p<0.0001)$, GGT $(p<0.0001)$, total bilirubin $(p=0.011)$, glycemia, $(p=0.0003)$ and cholesterol $(\mathrm{p}<0.0001)$ than CHC patients with F3-F4 (Table 1). CHC patients with F1-F2 had higher platelet count $(\mathrm{p}<0.0001)$, cholesterol $(\mathrm{p}<0.0001)$, and PT $(\mathrm{p}=0.002)$ than those with F3-F4 (Table 1$)$.

Differential hepatic miRNAs expression in patients with advanced fibrosis and cirrhosis. The hepatic expression of 13 miRNAs (miR-20a, miR-21, miR-27a, miR-27b, miR-29a, miR-29c, miR-92a, miR-122, miR-146a, miR-155, miR-221, miR-222, and miR-224) was compared between patients with F3-F4 and those with F1-F2 either in patients with CHB or CHC (Fig. 1 and Supplementary Figure 1).

In patients with $\mathrm{CHB}$, hepatic miR-122 ( $\mathrm{p}=0.004)$ (Fig. 1E) and miR-27b $(\mathrm{p}=0.038)$ (Supplementary Figure 1B) were decreased in patients with F3-F4 compared to those with F1-F2. Both miR-222 (p=0.018) (Fig. 1H) and miR-224 ( $\mathrm{p}=0.0001)$ (Fig. 1I) were increased in patients with F3-F4 compared to those with F1-F2 (Fig. 1).

In patients with $\mathrm{CHC}$, hepatic miR-122 was reduced in patients with F3-F4 compared to those with F1-F2 $(\mathrm{p}=0.008)($ Fig. $1 \mathrm{~N})$ and hepatic miR-224 was increased in patients with F3-F4 compared to those with F1-F2 $(\mathrm{p}=0.002)$ (Fig. 1R). The expression of hepatic miR-222 was not significantly different between patients with F3-F4 and those with F1-F2 (Fig. 1I).

Hepatic miR-20a, miR-21, miR-29a, miR-92a, miR-146a, miR-221 (Fig. 1A-D,F,J-M,O), miR-27a, miR29c and miR-155 (Supplementary Figure 1A, 1C-1H), showed no significant differences of expression between patients with F3-F4 and F1-F2 with either $\mathrm{CHB}$ or $\mathrm{CHC}$.

Differential serum miRNAs expression in patients with advanced fibrosis and cirrhosis. The serum expression of 13 miRNAs (miR-20a, miR-21, miR-27a, miR-27b, miR-29a, miR-29c, miR-92a, miR-122, miR-146a, miR-155, miR-221, miR-222, and miR-224) was compared to patients with CHB and CHC with F3-F4 and in those with F1-F2 (Fig. 2 and Supplementary Figure 2).

In patients with $\mathrm{CHB}$, the serum expression of miR-29a ( $\mathrm{p}=0.048)$, (Fig. $2 \mathrm{C})$ miR-92a $(\mathrm{p}=0.031)$ (Fig. 2D), and miR-122 ( $\mathrm{p}=0.049)$ (Fig. 2E) was reduced in patients with F3-F4 compared to those with F1-F2. Moreover, the expression of miR-146a $(\mathrm{p}=0.015)($ Fig. $2 \mathrm{~F})$ and miR-222 (Fig. $2 \mathrm{H})(\mathrm{p}=0.040)$ was increased in patients with F3-F4 compared to those with F1-F2. Serum expression of miR-20a, miR-21, miR-221 andmiR-224, miR-27a, miR-27b, miR-29c, miR-155 (Fig. 2A,B,G,Q, and Supplementary Figure 2A-D) showed no significant differences of expression between CHB patients with F3-F4 and those with F1-F2. 


\begin{tabular}{|c|c|c|c|c|c|c|c|c|c|c|}
\hline \multirow[b]{2}{*}{$\mathrm{N}$, patients } & \multicolumn{5}{|c|}{ Chronic hepatitis B } & \multicolumn{5}{|c|}{ Chronic Hepatitis C } \\
\hline & \begin{tabular}{|c|}
$\mathrm{N}$ \\
total $=58$
\end{tabular} & F0/F1/F2 & $\begin{array}{c}\mathrm{N} \\
\text { total }=44\end{array}$ & F3/F4 & p value & $\begin{array}{c}\mathrm{N} \\
\text { total }=84\end{array}$ & F0/F1/F2 & $\begin{array}{c}\mathrm{N} \\
\text { total }=94\end{array}$ & F3/F4 & p value \\
\hline Gender: male/female, n (\%) & 58 & $50(86.2) / 8(13.8)$ & 44 & $37(84.1) / 7(15.9)$ & 0.78 & 84 & $41(48.8) / 43(51.2)$ & 94 & $64(68.1) / 30(31.9)$ & 0.01 \\
\hline Age, years (median, IQ) & 58 & $42[30.25 ; 52.5]$ & 43 & $42[33.5 ; 47]$ & 0.83 & 84 & $49[43 ; 55]$ & 92 & $49[44 ; 54]$ & 0.94 \\
\hline BMI, kg.m ${ }^{-2}$ (median, IQ) & 50 & $25.1[23.1 ; 27.5]$ & 41 & $25.9[23.2 ; 28.3]$ & 0.43 & 70 & $24.6[22.2 ; 27.8]$ & 64 & $25.8[24.2 ; 28.8]$ & 0.045 \\
\hline ALT, IU/L (median, IQ) & 58 & $66[49 ; 120.8]$ & 44 & $78.5[54.8 ; 161]$ & 0.50 & 82 & $79.5[57.2 ; 101.5]$ & 92 & $119[65.5 ; 154.5]$ & 0.0002 \\
\hline AST, IU/L (median, IQ) & 58 & $40.5[34.3 ; 62.8]$ & 44 & $59.5[36 ; 131]$ & 0.047 & 82 & $47[36.5 ; 62.8]$ & 91 & $77[52 ; 112]$ & $<0.0001$ \\
\hline ALP, IU/L (median, IQ) & 57 & $43[27 ; 63]$ & 40 & $94[44.5 ; 181.5]$ & 0.0002 & 82 & $65[52 ; 78.8]$ & 88 & $80[60.8 ; 107]$ & $<0.0001$ \\
\hline GGT, IU/L (median, IQ) & 55 & $15[11 ; 17]$ & 43 & $18[14.5 ; 24.5]$ & 0.001 & 82 & $44.5[26 ; 78.5]$ & 90 & $96[58.25 ; 156]$ & $<0.0001$ \\
\hline Platelets, $\mathrm{x} 10^{3} / \mathrm{mm}^{3}($ median, IQ) & 58 & $196.5[162 ; 234.8]$ & 43 & $156[135 ; 192]$ & 0.0003 & 78 & $230[188 ; 263]$ & 83 & $172[120.5 ; 209]$ & $<0.0001$ \\
\hline Cholesterol, mmol/L (median, IQ) & & NA & & $\mathrm{NA}$ & & 68 & $4.75[4.3 ; 5.5]$ & 60 & $4.2[3.6 ; 4.7]$ & $<0.0001$ \\
\hline Triglycerides, mmol/L (median, IQ) & 50 & $0.9[0.6 ; 1.1]$ & 32 & $1.0[0.7 ; 1.7]$ & 0.035 & 67 & $0.94[0.7 ; 1.1]$ & 59 & $0.92[0.7 ; 1.4]$ & 0.44 \\
\hline Glycemia, mmol/L (median, IQ) & 51 & $4.8[4.3 ; 5.1]$ & 35 & $5.1[4.5 ; 5.9]$ & 0.027 & 71 & $4.9[4.5 ; 5.4]$ & 71 & $5.4[4.8 ; 6.1]$ & 0.0003 \\
\hline Total bilirubin, $\mu \mathrm{mol} / \mathrm{L}$ (median, IQ) & 22 & $10.1[6.3 ; 13.8]$ & 6 & $16,9[13.6 ; 39.5]$ & 0.031 & 79 & $12[9 ; 15]$ & 90 & $14[10 ; 18]$ & 0.01 \\
\hline Albumin, g/L (median, IQ) & 57 & $47.2[44 ; 49.4]$ & 41 & $44.4[41.4 ; 47.3]$ & 0.002 & 69 & $45.4[44 ; 48]$ & 70 & $45.1[43 ; 47.8]$ & 0.26 \\
\hline Viral Loads, $\log \mathrm{UI} / \mathrm{mL}$ (median, IQ) & 58 & $6.2[4.3 ; 7.3]$ & 42 & $5.9[4.1 ; 7.2]$ & 0.27 & 52 & $5.6[5.2 ; 6.1]$ & 59 & $5.9[5.3 ; 6.3]$ & 0.10 \\
\hline Necroinflammatory activity, n (\%) & 58 & & 44 & & 0.016 & 83 & & 89 & & 0.001 \\
\hline None $(A 0)$ & & $4(6.9)$ & & $1(2.3)$ & & & $10(11.9)$ & & $5(5.3)$ & \\
\hline Mild (A1) & & $33(56.9)$ & & $18(40.9)$ & & & $58(69.0)$ & & $44(46.8)$ & \\
\hline Moderate (A2) & & $20(34.5)$ & & $20(45.4)$ & & & $14(16.7)$ & & $35(37.2)$ & \\
\hline Severe (A3) & & $1(1.7)$ & & $5(11.4)$ & & & $1(1.2)$ & & $5(5.3)$ & \\
\hline NA & & 0 & & 0 & & & $1(1.2)$ & & $5(5.3)$ & \\
\hline Steatosis Grades, n (\%) & 58 & & 44 & & 0.26 & 82 & & 84 & & 0.02 \\
\hline 0 & & $35(60.3)$ & & $22(50)$ & & & $37(44.05)$ & & $23(24.5)$ & \\
\hline 1 & & $15(25.9)$ & & $12(27.3)$ & & & $27(32.14)$ & & $24(25.5)$ & \\
\hline 2 & & $5(8.6)$ & & $8(18.1)$ & & & $15(17.86)$ & & $28(29.8)$ & \\
\hline 3 & & $0(0)$ & & $1(2.3)$ & & & $3(3.57)$ & & $9(9.6)$ & \\
\hline $\mathrm{NA}$ & & $3(5.2)$ & & $1(2.3)$ & & & $2(2.38)$ & & $10(10.6)$ & \\
\hline HCV genotypes, n (\%) & & - & & - & & 78 & & 88 & & 0.007 \\
\hline 1 & & - & & - & & & $40(47.6)$ & & $48(51.1)$ & \\
\hline 2 & & - & & - & & & $11(13.1)$ & & $2(2.1)$ & \\
\hline 3 & & - & & - & & & $11(13.1)$ & & $17(18.1)$ & \\
\hline 4 & & - & & - & & & $11(13.1)$ & & $21(22.3)$ & \\
\hline 5 & & - & & - & & & $3(3.6)$ & & $0(0)$ & \\
\hline 6 & & - & & - & & & $1(1.2)$ & & $0(0)$ & \\
\hline $\mathrm{NA}$ & & - & & - & & & $7(8.3)$ & & $6(6.4)$ & \\
\hline
\end{tabular}

Table 1. Characteristics of the patients according to the etiology of the liver disease (CHB and CHC). AST, aspartate aminotransferase; ALT, alanine aminotransferase; ALP, alkaline phosphate; BMI, Body mass index; GGT, Gamma-glutamyltranspeptidase, IQ: Interquartile range, NA: Not available. Clinical parameters are expressed as median and interquartile range (IQ), unless indicated differentially. Differences between chronic hepatitis B and C patients were evaluated with the Fisher's exact (qualitative variables) and the Wilcoxon ranksum tests (continuous variables).

MiR-29a, -92a, -122, -146a and -222 were all deregulated in patients with F3-F4 compared to those with F0-F2. Therefore the expression of these 5 miRNAs was tested in a group of seven patients with CHB who started nucleosides/nucleotides inhibitors (Nucs) therapy to abrogate HBV replication. One serum was available before the initiation of treatment and a second one 12.5 months (IQ: 11.5-14) after the initiation of Nucs (Table S3). In agreement with expectations ${ }^{23}$, serum HBV DNA was undetectable at the time of the second serum for the 7 patients (Table S3). None of the miRNAs tested (eg. miR-29a, -92a, -122, -146a and -222) was differentially expressed after abrogation of HBV replication following one year of Nucs therapy (Supplementary Figure 3A-E).

In patients with $\mathrm{CHC}$, the serum expression of miR-20a, miR-21, miR-29a, miR-92a, miR-122, miR-146a, miR-221, miR-222, and miR-224 (Fig. 2J-R) showed no difference in patients with F3-F4 when compared to those with F1-F2 (Fig. 2). The level of expression of serum miR-27a, miR-27b, miR-29c and miR-155 was under the limit of detection in patients with CHC (data not shown). In CHC patients since none of the miRNAs tested was significantly deregulated in serum, we followed the expression of the miRNAs that were deregulated in the liver and/or those almost significantly differentially expressed in serum samples of patients with F3-F4 compared to those with F0-F1-F2. (eg. MiR-122, -221, -222 and -224) in 9 patients before and after sustained virological response following IFN-based treatment $(n=8)$ or DAAs $(n=1)$. Sustained virological response is defined 


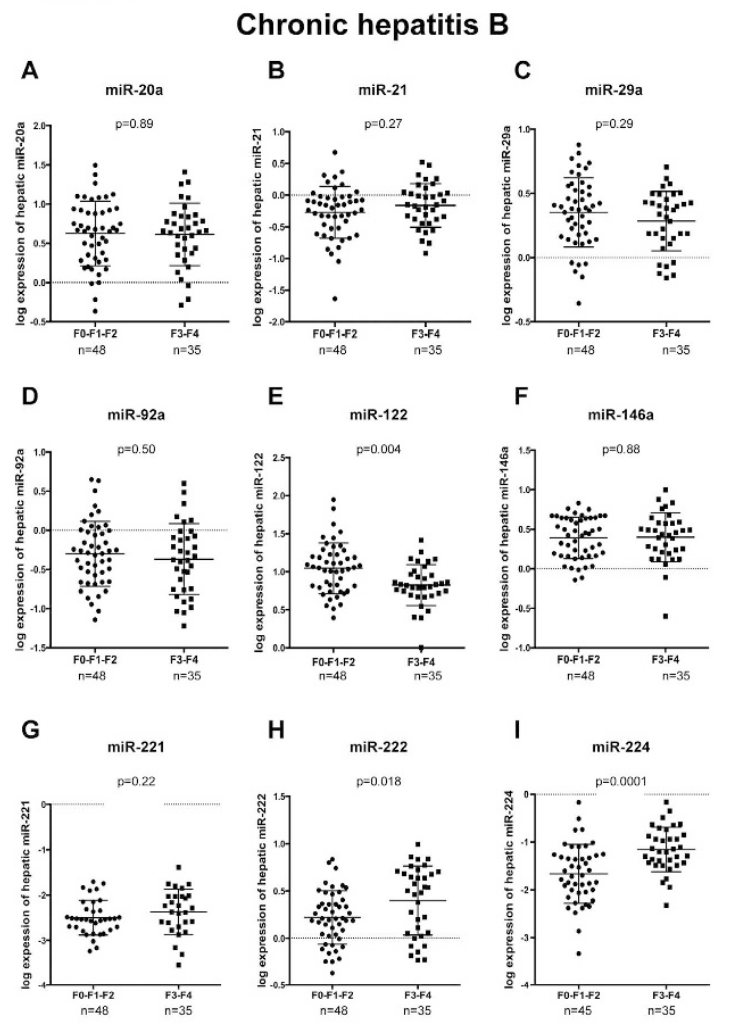

\section{Chronic hepatitis C}
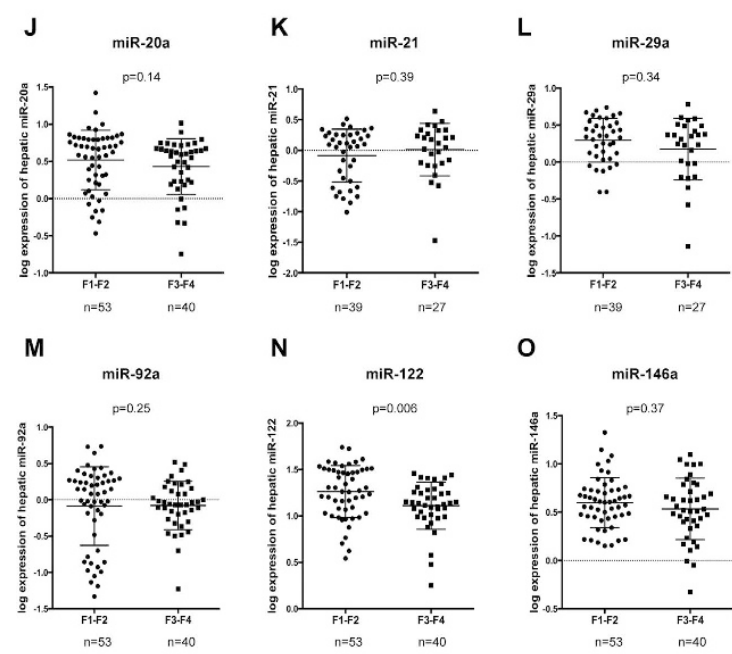

○
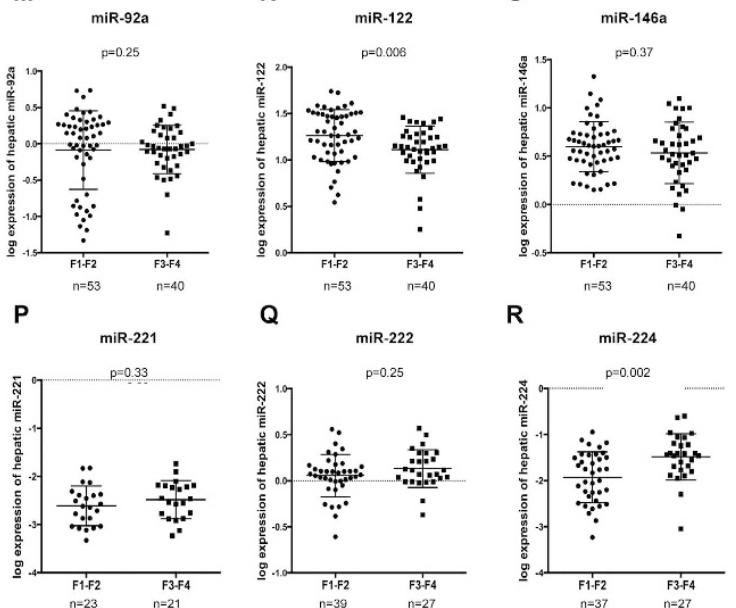

R

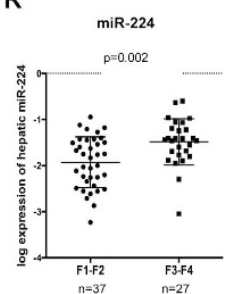

Figure 1. Differences in the expression of hepatic miRNAs according to the stage of fibrosis in patients with chronic hepatitis B and C. The expression of hepatic miR-20a, miR-21, miR-29a, miR-92a, miR-122, miR-146a, miR-221, miR-222, miR-224 was assessed by RT-qPCR from $1 \mathrm{ng}$ of cDNA and compared in patients with F3-F4 and F0-F1-F2, in chronic hepatitis B patients (A-I) and in those with chronic hepatitis $\mathrm{C}(\mathbf{J}-\mathbf{R})$. The $\Delta \mathrm{Ct}$ $\left(\Delta \mathrm{Ct}=2^{\Delta \mathrm{C} \text {,sample }}\right.$ ) of each miRNA was calculated and normalized to the $\Delta \mathrm{Ct}$ value of SNORD44 in each biopsy. The log expression of the ratio miRNA/SNORD44 is shown as dot plot, each dot represents one patient (mean and standard deviation). The Wilcoxon rank-sum test was used to compare miRNAs expression. 


\section{Chronic hepatitis B}
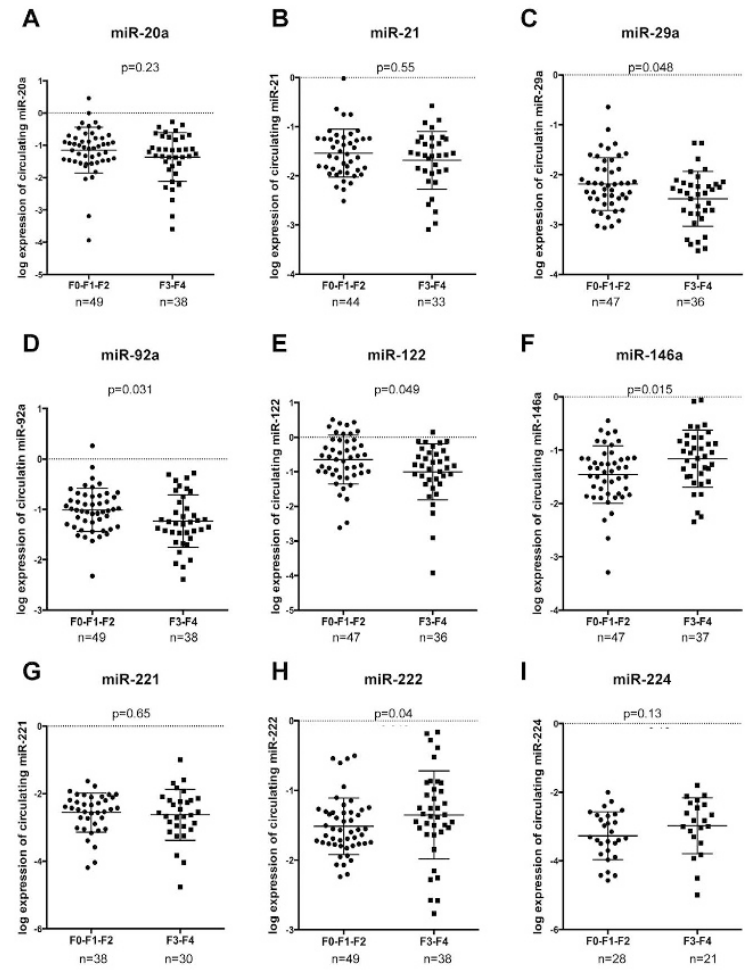

\section{Chronic hepatitis C}
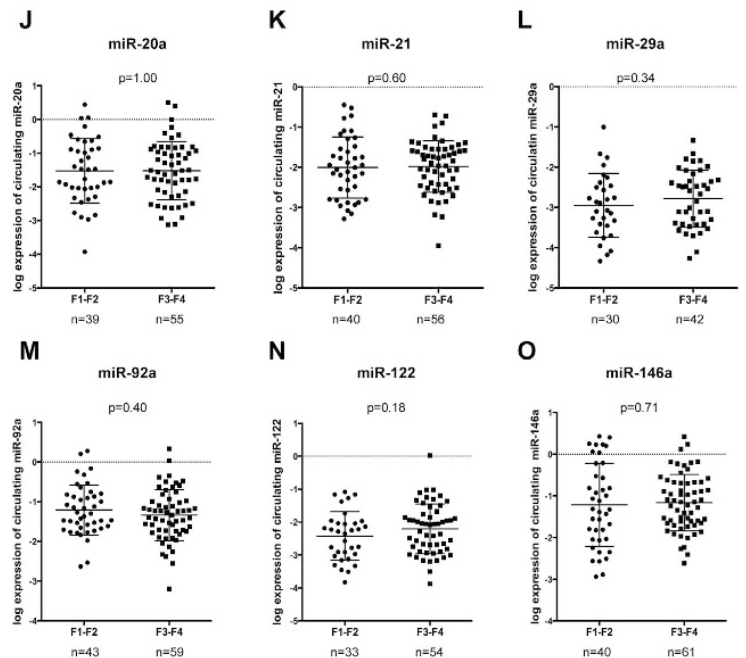

N
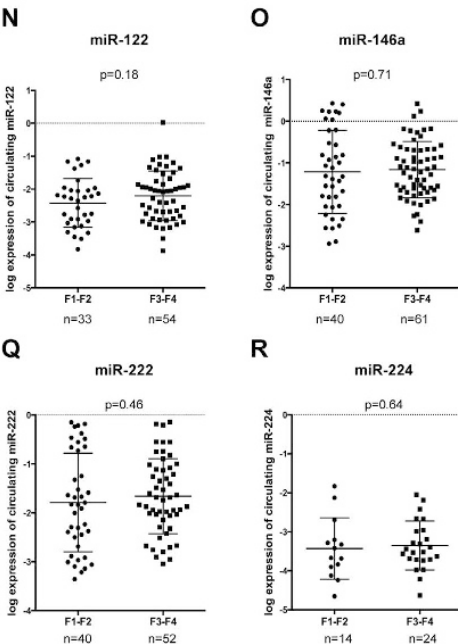

Figure 2. Differences of the expression of serum miRNAs according to the stages of fibrosis in patients with chronic hepatitis B and C. The expression of serum miR-20a, miR-21, miR-29a, miR-92a, miR-122, miR-146a, miR-221, miR-222, miR-224 was assessed by RT-qPCR and compared in patients with F3-F4 and F0-F1-F2, in chronic hepatitis B patients $(\mathbf{A}-\mathbf{I})$ and in those with chronic hepatitis $\mathrm{C}(\mathbf{J}-\mathbf{R})$. The $\Delta \mathrm{Ct}\left(\Delta \mathrm{Ct}=2^{\Delta \mathrm{Cp} \text {,sample }}\right)$ of each miRNA was calculated and normalized to the $\Delta$ Ct value of the exogenous C. elegans-miR-39 (cel-miR-39) in each serum. The log expression of the ratio miRNA/cel-miR-39 is shown as dot plot, each dot represents one patient (mean and standard deviation). The Wilcoxon rank-sum test was used to compare miRNAs expression. 


\begin{tabular}{|l|c|c|c|c|}
\hline & & OR & 95\% CI & p value \\
\hline \multirow{4}{*}{ CHB patients } & Serum miR-122 & 0.19 & $0.03-1.17$ & 0.07 \\
\cline { 2 - 5 } & Serum miR-222 (x100) & 1.12 & $1.01-1.24$ & 0.03 \\
\cline { 2 - 5 } & Platelets & 0.99 & $0.98-1.00$ & 0.09 \\
\cline { 2 - 5 } & ALP $(/ 10)$ & 1.21 & $1.04-1.41$ & 0.01 \\
\hline \multirow{5}{*}{ CHC patients } & Hepatic miR-122 & 0.80 & $0.67-0.95$ & 0.01 \\
\cline { 2 - 5 } & Hepatic miR-224 (x100) & 1.56 & $1.05-2.31$ & 0.03 \\
\cline { 2 - 5 } & Platelets & 0.97 & $0.95-0.99$ & 0.004 \\
\cline { 2 - 5 } & ALT (/10) & 1.09 & $0.96-1.24$ & 0.17 \\
\cline { 2 - 5 } & Albumin & 1.41 & $0.94-2.11$ & 0.10 \\
\hline
\end{tabular}

Table 2. Multivariate analysis of clinical data and serum microRNA and their association with fibrosis in patients with $\mathrm{CHB}$ and $\mathrm{CHC}$.

as HCV RNA undetectable 12 weeks after the end of treatment ${ }^{24}$. For these 9 patients the second serum was available 12.0 (IQ: 9-13) months after the end of the treatment (Table S3). None of these miRNAs was differentially expressed after HCV eradication (Supplementary Figure 3G-I). However, a more detailed analysis of fibrosis-associated miRNAs in treated $\mathrm{CHB}$ and $\mathrm{CHC}$ patients may inform on the recovery and/or regression of fibrosis while avoiding liver biopsy.

MiRNAs-based signature in patients with $\mathrm{CHB}$ and comparison of its diagnostic performance with APRI and FIB-4. In univariate analysis, all the following variables (aspartate aminotransferase (AST), alanine aminotransferase (ALT), alkaline phosphate (ALP), albumin, platelet count, serum miR-29a, miR-92a, miR-122, miR-146a and miR-222) were associated with F3-F4 (Fig. 2). The characteristics of the patients for whom serum samples were available were used for the establishment of the CHB model (Table S4). Serum miR$222(\mathrm{OR}=1.12 ; 95 \% \mathrm{CI}: 1.01-1.24 ; \mathrm{p}=0.03)$ and $\mathrm{ALP} \mathrm{OR}=1.21 ; 95 \% \mathrm{CI}: 1.04-1.41 ; \mathrm{p}=0.02)$ levels were independently associated with stages of fibrosis (Table 2). Combined together, serum miR-122, serum miR-222, ALP and platelet count were the strongest associated predictors of advanced fibrosis and cirrhosis (F3-F4) (Table 2). ROC analysis was performed to evaluate the diagnostic performances of the model combining serum miR-122, serum miR-222, platelet count and ALP levels to discriminate patients with F3-F4 from those with F1-F2.

The model combining serum miR-122, serum miR-222, platelet count and ALP (called CHB model) discriminates patients with F3-F4 from those with F1-F2 with an AUC of 0.86 ( $95 \% \mathrm{CI}=0.77-0.95)$ (Table 3). This CHB model was more accurate than FIB-4 (AUC $=0.81 ; 95 \% \mathrm{CI}=0.71-0.90)$ and APRI $(\mathrm{AUC}=0.70 ; 95 \% \mathrm{CI}=0.58-0.82)$ to discriminate patients with F3-F4 from those with F1-F2 (Table 3). The CHB model was statistically better than APRI $(p=0.007)$ but not than FIB-4 $(p=0.39)$ (Table 3$)$. By optimizing with the Youden's index, the optimal cut-off of the CHB model for the diagnosis of patients with F3-F4 was: 0.57 (sensitivity $(\mathrm{Se})=0.79$, specificity $(\mathrm{Sp})=0.95, \mathrm{PPV}=0.79, \mathrm{NPV}=0.95)($ Table 4$)$.

To optimally discriminate between patients with F3-F4 and those with F1-F2 both APRI and FIB-4 need 2 different cut-offs ( $<0.5$ and $>1.5$ for APRI and $<1.45$ and $>3.25$ for FIB- 4 ).

Taking into account the cut-offs to differentiate patients with advanced fibrosis and cirrhosis (F3-F4) from patients with mild-moderate fibrosis (F1-F2), the sensitivity of the $\mathrm{CHB}$ model $(\mathrm{Se}=0.79)$ was respectively higher and lower than the one of FIB-4 $(\mathrm{Se}=0.67)$ and the one of APRI $(\mathrm{Se}=0.84)$ (Table 4). Moreover, the specificity of our CHB model $(\mathrm{Sp}=0.95)$ was higher than the one of APRI $(\mathrm{Sp}=0.90)$ and lower than the one of FIB-4 $(\mathrm{Sp}=0.97)$ (Table 4$)$.

The positive predictive value $(\mathrm{PPV})$ of the $\mathrm{CHB}$ model $(\mathrm{PPV}=0.79)$ was higher than the one of APRI $(\mathrm{PPV}=0.27$ for the 0.5 cut-off and PPV $=0.46$ for the 3.25 cut-off) and FIB- 4 (PPV $=0.38$ for the 1.45 cut-off and 0.65 for 1.5 cut-off) (Table 4$)$.

The negative predictive value (NPV) of our CHB model (NPV $=0.95)$ was higher than the ones of FIB-4 $(\mathrm{NPV}=0.90$ for the 1.45 cut-off and 0.84 for 3.25 cut-off) and APRI (NPR $=0.91$ for the 0.5 cut-off and 0.85 for the 1.5 cut-off) (Table 4$)$.

Respectively $32(14+18)$ and $28(7+21)$ patients of the 44 patients with F3-F4 had a FIB- $4<3.25$ and an APRI $<1.5$ that may account for relatively low PPV of APRI and FIB-4 in our cohort of patients (Tables S5 and S6).

MiRNAs-based signature in patients with $\mathrm{CHC}$ and comparison of its diagnostic performance with APRI and FIB-4. In univariate analysis, albumin, platelet count, AST, ALT, ALP, hepatic miR-122 and miR-224 were all associated with F3-F4. Therefore, these variables were considered for the model selection of the multivariate analysis. The characteristics of the patients for whom liver samples were available were used for the establishment of the CHC model (Table S7). The strongest independently associated predictors of F3-F4 were hepatic miR-122 $(p=0.01)$, hepatic miR-224 $(p=0.03)$ and platelets count $(p=0.004)$ (Table 2$)$. ROC analysis was performed to evaluate the diagnostic performances of the model to discriminate patients with F3-F4 from those with F1-F2.

The model combining hepatic miR-122, hepatic miR-224, platelets count, ALT and albumin (defined as the $\mathrm{CHC}$ model) was the strongest one to discriminate patients with F3-F4 from those with F1-F2 with an AUC of $0.93(95 \%, \mathrm{CI}=0.83-0.87)$. The $\mathrm{CHC}$ model was more accurate than both FIB-4 (AUC $=0.79,95 \% \mathrm{CI}=0.67-$ 0.90 ) and APRI ( $\mathrm{AUC}=0.86,95 \% \mathrm{CI}=0.75-0.96)$ to discriminate F3-F4 from F1-F2 (Table 3). The CHC model was statistically better than FIB-4 $(\mathrm{p}=0.009)$ to discriminate patients with F3-F4 from those with F1-F2 (Table 3). 


\begin{tabular}{|c|c|c|c|}
\hline CHB model & AUC & $95 \%$ CI & p value \\
\hline Serum miR-122 & \multirow{4}{*}{0.85} & \multirow{4}{*}{$0.77-0.95$} & \\
\hline Serum miR-222 & & & \\
\hline Platelet count & & & \\
\hline ALP & & & \\
\hline APRI & 0.70 & $0.58-0.82$ & 0.007 \\
\hline FIB-4 & 0.81 & $0.71-0.90$ & 0.39 \\
\hline Hepatic miR-122 & \multirow{5}{*}{0.93} & \multirow{5}{*}{$0.87-0.99$} & \\
\hline Hepatic miR-224 & & & \\
\hline Platelet count & & & \\
\hline ALT & & & \\
\hline Albumin & & & \\
\hline APRI & 0.86 & $0.75-0.96$ & 0.11 \\
\hline FIB-4 & 0.79 & $0.67-0.90$ & 0.009 \\
\hline
\end{tabular}

Table 3. Diagnostic performance of the miRNAs-based models to diagnose F3-F4 fibrosis in patients with CHB and CHC.

\begin{tabular}{|c|c|c|c|c|c|c|}
\hline Etiology & Score & $\begin{array}{c}\text { Cut-off } \\
\text { value }\end{array}$ & $\begin{array}{l}\text { Sensitivity } \\
(95 \% \mathrm{CI})\end{array}$ & $\begin{array}{c}\text { Specificity } \\
(95 \% \mathrm{CI})\end{array}$ & PPV & NPV \\
\hline \multirow{5}{*}{$\mathrm{CHB}$ patients } & FIB-4 & 1.45 & $0.67(0.51-0.79)$ & $0.72(0.60-0.83)$ & 0.38 & 0.90 \\
\hline & & 3.25 & $0.26(0.14-0.4)$ & $0.97(0.91-1)$ & 0.65 & 0.84 \\
\hline & APRI & 0.5 & $0.84(0.72-0.93)$ & $0.43(0.31-0.55)$ & 0.27 & 0.91 \\
\hline & & 1.5 & $0.35(0.21-0.49)$ & $0.9(0.81-0.97)$ & 0.46 & 0.85 \\
\hline & $\begin{array}{c}\text { Our miRNA- } \\
\text { based signature }\end{array}$ & 0.57 & $0.79(0.71-0.88)$ & $0.95(0.92-0.97)$ & 0.79 & 0.95 \\
\hline \multirow{5}{*}{ CHC patients } & FIB-4 & 1.45 & $0.76(0.66-0.85)$ & $0.67(0.56-0.77)$ & 0.36 & 0.92 \\
\hline & & 3.25 & $0.27(0.18-0.37)$ & $0.99(0.96-1)$ & 0.84 & 0.84 \\
\hline & APRI & 0.5 & $0.91(0.85-0.98)$ & $0.4(0.29-0.51)$ & 0.27 & 0.95 \\
\hline & & 1.5 & $0.38(0.28-0.48)$ & $0.95(0.90-0.99)$ & 0.65 & 0.86 \\
\hline & $\begin{array}{c}\text { Our miRNA- } \\
\text { based signature }\end{array}$ & 0.32 & $0.89(0.86-0.92)$ & $0.84(0.80-0.88)$ & 0.58 & 0.97 \\
\hline
\end{tabular}

Table 4. Analytical performance of APRI, FIB-4 and our miRNA-based models to diagnose fibrosis in CHB and CHC patients. A cut-off value for the prediction of patients with F3-F4 fibrosis was determined by maximizing the Youden index. The optimal cut-off values were assessed by the sensitivity, specificity, positive (PPV) and negative predictive values (NPV). PPV and NPV were calculated as follow: (Prevalence of patients with F3-F4 among the total population of patients with viral hepatitis is: $20 \%) . \mathrm{PPV}=[$ sensitivity $\times$ prevalence]/ $[$ sensitivity $\times$ prevalence $+(1-$ specificity $) \times(1-$ prevalence $)] . N P V=[$ specificity $\times(1-$ prevalence $)] /$ $[(1-$ sensitivity $) \times$ prevalence + specificity $\times(1-$ prevalence $)]$.

As for the CHB model, a single cut-off of 0.32 was defined for the $\mathrm{CHC}$ model by optimizing with the Youden's index (Table 4). Therefore, there was no grey zone in the calculation of $\mathrm{CHB}$ and $\mathrm{CHC}$ value and the fibrosis was predictable for all the patients.

Taking into account the two different cut-offs to differentiate patients with F3-F4 from those with F1-F2 for both APRI and FIB-4, the sensitivity of the CHC model (0.89) was higher than the one of FIB-4 $(\mathrm{Se}=0.76)$ and lower than the one of APRI $(\mathrm{Se}=0.91)$ (Table 4$)$.

The specificity of the CHC model $(\mathrm{Sp}=0.84)$ was lower than the ones of FIB- 4 ( $\mathrm{Sp}=0.99$ with the cut-off of 3.25) and APRI (0.95 with the cut-off of 1.5) (Table 4).

The PPV of our CHC model (PPV $=0.58$ ) was lower than the ones of APRI (PPV $=0.84$ for the cut-off of 3.25) and FIB-4 (0.65 for the cut-off of 1.5) (Table 4).

The NPV of the CHC model $(\mathrm{NPV}=0.97)$ was higher than the ones of APRI ( 0.95 for the cut-off of 0.5 and 0.86 for cut-off of 1.5) and FIB-4 (0.92 for the cut-off of 0.5 and 0.84 for the cut-off of 1.5) (Table 13) irrespective of the cut-off values. Respectively $58(19+39)$ and $50(7+43)$ patients of 94 patients with F3-F4 had a FIB- $4<3.25$ and an APRI $<0.5$ that may account for relatively low PPV of APRI and FIB- 4 in our cohort of patients (Tables S5 and S6).

Differential miRNAs expression between patients with CHB and CHC. Hepatic miR-20a $(p=0.052)$, miR-29a $(p=0.35), \operatorname{miR}-29 c(p=0.17)$ and miR-221 $(p=0.17)$ were expressed in an equivalent way in the liver of patients with CHC and CHB (Figure). However, hepatic miR-21 ( $\mathrm{p}=0.001), \operatorname{miR}-92 \mathrm{a}(\mathrm{p}<0.0001)$, miR-122 ( $\mathrm{p}<0.0001)$, miR-146a $(\mathrm{p}=0.0002), \operatorname{miR}-27 \mathrm{a}(\mathrm{p}<0.0001), \operatorname{miR}-27 \mathrm{~b}(\mathrm{p}=0.0002)$ and $\mathrm{miR}-155$ $(\mathrm{p}<0.0001)$ were increased in the liver of patients with $\mathrm{CHC}$ as compared to those with CHB (Fig. 3B,D-F and 
Supplementary Figure 4A,B,D). Interestingly, the level of expression of hepatic miR-122 in patients with CHC was 1.96-fold higher than in patients with CHB, independently of the stage of fibrosis (Fig. 3). Hepatic miR-222 and miR-224 were decreased in patients with CHC compared to those with CHB.

Serum miR-146a $(\mathrm{p}=0.31)$, miR-221 $(\mathrm{p}=0.35)$, and miR-224 $(\mathrm{p}=0.057)$ were expressed in an equivalent way in the serum of patients with CHB and CHC (Fig. 3). However, serum miR-20a $(p=0.010), \operatorname{miR}-21(p<0.0001)$, miR-29a $(\mathrm{p}<0.0001)$, miR-92a $(\mathrm{p}=0.017)$ miR-122 $(\mathrm{p}<0.0001)$ and miR-222 $(\mathrm{p}=0.008)$ were decreased in serums of patients with $\mathrm{CHC}$ compared to those with $\mathrm{CHB}$ (Fig. 3). Interestingly, the level of expression of serum miR-122 was 28 -fold higher in patients with $\mathrm{CHB}$ than in those with $\mathrm{CHC}$, independently of the stage of fibrosis.

Correlations between serum and hepatic miRNAs expression. In order to assess if serum miRNAs levels can reflect the level of hepatic miRNAs, we analyzed the correlation between the level of serum miRNAs and the level of hepatic miRNAs in patients with $\mathrm{CHB}$ and $\mathrm{CHC}$.

Hepatic and serum miR-20a $\left(R^{2}=0.31, p=0.01\right)$ and miR-146a $\left(R^{2}=0.36, p=0.009\right)$ expression were correlated. No correlation was observed for other miRNAs (Table S8). An inversed correlation was observed between hepatic miR-20a $\left(R^{2}=-0.31 ; \mathrm{p}\right.$-value $\left.=0.01\right)$, miR-92a $\left(\mathrm{R}^{2}=-0.25 ; \mathrm{p}\right.$-value $\left.=0.02\right)$, and $\mathrm{miR}-122\left(\mathrm{R}^{2}=-0.25\right.$; $\mathrm{p}$-value $=0.01)$ and ALT (Table S9). An inversed correlation was observed between hepatic miR-20a $\left(\mathrm{R}^{2}=-0.36\right.$; $\mathrm{p}$-value $=0.01)$, miR-92a $\left(\mathrm{R}^{2}=-0.27 ; \mathrm{p}\right.$-value $\left.=0.01\right)$, and miR-122 $\left(\mathrm{R}^{2}=-0.35 ; \mathrm{p}\right.$-value $\left.=0.01\right)$, and AST $($ Table S9). No correlation was observed between the other studied miRNAs and AST and ALT (Data not shown).

Correlations between the expression of miRNAs and ALT and AST. In the CHB cohort, serum miR122 was correlated with ALT $\left(\mathrm{R}^{2}=0.28, \mathrm{p}=0.01\right)$ and serum miR-21 was positively correlated with both ALT $\left(\mathrm{R}^{2}=0.23, \mathrm{p}=0.04\right)$ and AST $\left(\mathrm{R}^{2}=0.23, \mathrm{p}=0.04\right)($ Table 5$)$.

In the CHC cohort, serum miR-122 was positively correlated with the levels of both ALT $\left(\mathrm{R}^{2}=0.28, \mathrm{p}=0.01\right)$ and AST $\left(R^{2}=0.31, p=0.009\right)$. No significant correlations were reported between the expression of the others serum miRNAs and the level of ALT and AST (Table 5).

\section{Discussion}

The major novelty of our work consists in the identification of two models based on the expression of miRNAs designed to diagnose advanced fibrosis and cirrhosis (F3-F4) in patients with CHB and CHC. To develop these models, we report differential profiles of expression of miRNAs in the liver and serum of patients with $\mathrm{CHB}$ and $\mathrm{CHC}$.

The non-invasive model combining serum miR-122, serum miR-222, platelet count and alkaline phosphatase (ALP) for CHB patients allowed the identification of patients with F3-F4 from those with F1-F2. Interestingly, the CHB model had higher specificity than APRI ( 0.95 vs 0.90$)$ and a higher sensitivity than FIB-4 (0.79 vs 0.67$)$ (Table 4). Moreover with a single cut-off, this model was suitable to identify advanced fibrosis and cirrhosis (F3-F4) in all the patients tested. Overall, the CHB model was more accurate than APRI and FIB-4 to stage fibrosis (F3-F4 vs F1-F2) in all patients with $\mathrm{CHB}(\mathrm{Se}=0.79, \mathrm{Sp}=0.95, \mathrm{PPV}=0.79, \mathrm{NPV}=0.95)$ (Table 4). Whereas, the CHB model may help to monitor fibrosis for both positive and negative HBeAg carriers, it should be validated in a larger cohort of patients to be used in clinical practice.

The reduction of hepatic and serum miR-122 in CHB patients (Figs 1 and 2) with F3-F4 is in agreement with previous reports ${ }^{18,20}$. Serum miR-122 is a relevant biomarker in liver disease ${ }^{25,26}$. Indeed, the assessment of serum miR-122, miR-21 and miR-29c discriminates patients with cirrhosis (F4) among patients with $\mathrm{CHB}^{18}$.

The expression of both serum and hepatic miR-222 was increased in CHB patients with F3-F4 compared to those with F1-F2 (Figs 1 and 2). MiR-222 promotes the proliferation and differenciation of hepatic stellate cells (HSCs) ${ }^{27}$. Moreover, miR-222 downregulates the expression of ICAM- $1^{28}$ which is responsible for the transmigration of immune cells to the sites of liver damage ${ }^{29}$. Interestingly, the up-regulation of miR-222 in F3-F4 patients may accelerate the activation of HSCs, increases the inflammation in the liver and thus facilitates the progression of fibrosis.

Although the World Health Organization recommended the use of APRI to detect significant fibrosis and cirrhosis (F2-F3-F4) in resource-limited countries ${ }^{15,16}$ its accuracy and sensitivity remain moderate to discriminate in between the stages of fibrosis in patients with $\mathrm{CHB}^{10,14}$. FIB-4 is suggested to be superior to APRI to diagnose cirrhosis in patients with $\mathrm{CHB}^{30-32}$. However, overall because of the weakness of their diagnostic performance, APRI and FIB- 4 do not seem to be suitable for use in clinical practice in patients with $\mathrm{CHB}^{10,14}$. Other non-invasive tests have been developed to stage fibrosis in patients with CHB. The combination of AST, GGT, AFP and platelet count was able to diagnose severe fibrosis and cirrhosis (F3-F4) in CHB patients with an AUC of $0.85^{33}$. The Platelet count/Age/ALP/Alpha-foeto-protein/AST (PAPAS) index was able to diagnose significant fibrosis (F2-F3-F4) with an AUC of 0.776 and a NPV of $88.4 \%{ }^{34}$. Interestingly, these two last scores take both into account the level of AST that is not always associated with histological lesions in CHB patients ${ }^{35}$. Likewise PAPAS index, the non-invasive miRNAs-based model for patients with CHB includes ALP and platelet count, suggesting that those two biomarkers are particularly relevant to diagnose fibrosis. More recently, the model including GGT to platelet ratio (GPR) developed in patients with $\mathrm{CHB}$ was less accurate than APRI and FIB-4 to identify significant fibrosis (F2-F3-F4) and cirrhosis ${ }^{36}$.

The CHC model combining hepatic miR-122, hepatic miR-224, platelet count, ALT and albumin allowed the identification of $\mathrm{CHC}$ patients with F3-F4 from those with F1-F2 with better diagnostic performances than APRI and FIB-4 (AUC $=0.93,95 \% \mathrm{CI}=0.87-0.99, \mathrm{Se}=0.89, \mathrm{Sp}=0.84, \mathrm{NPV}=0.97$ ) (Table 4). Since this model takes into account hepatic expression of miR-122 and miR-224, its use may be limited compared to APRI and FIB-4. However, a risk of miss-classification of $20 \%$ is associated with liver histology staging ${ }^{7,37,38}$. Therefore, the quantification of hepatic miR-122 and miR-224 in patients with $\mathrm{CHC}$ who undergo liver biopsy may help to better characterize the stage of fibrosis and reduce the risk of mis-classification. 

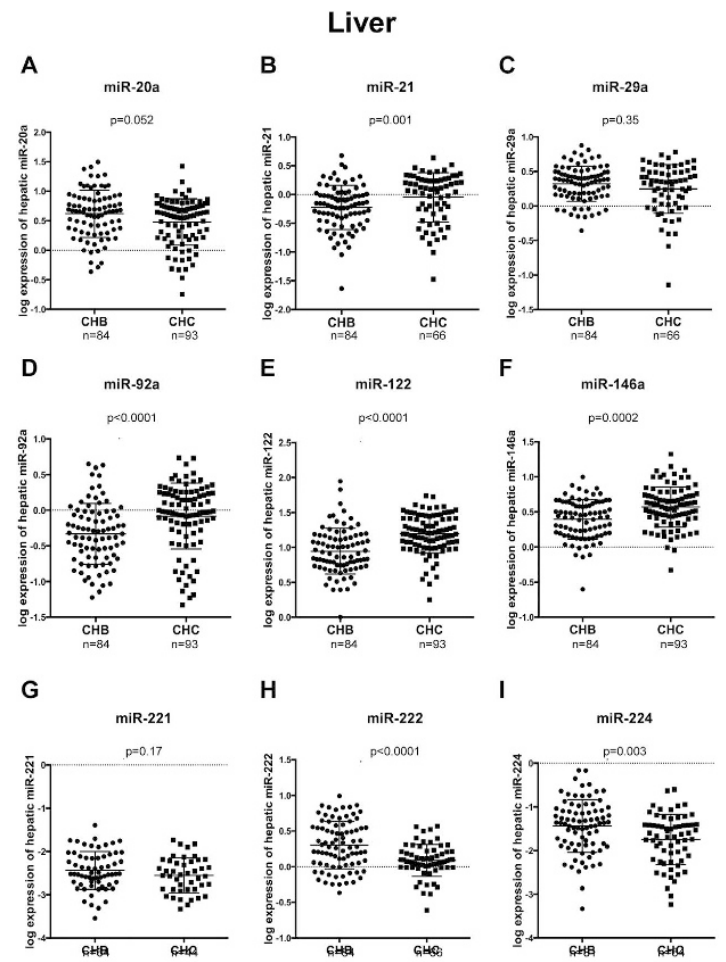

Serums
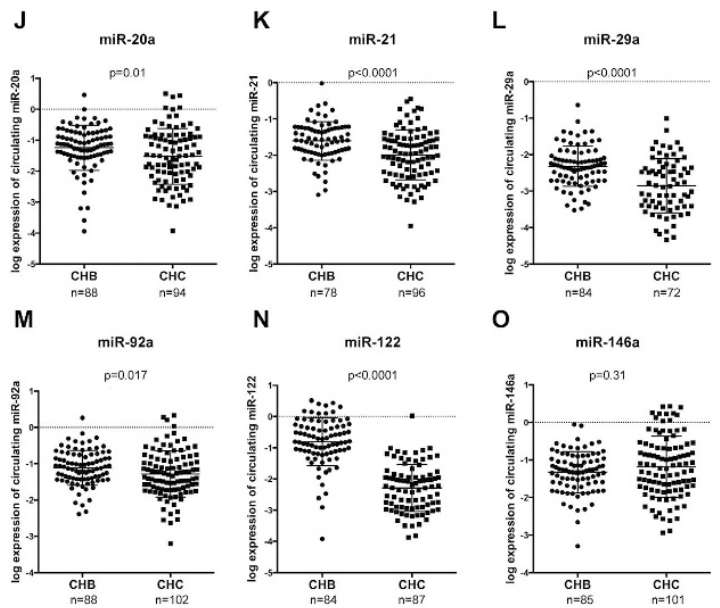

$\mathbf{N}$

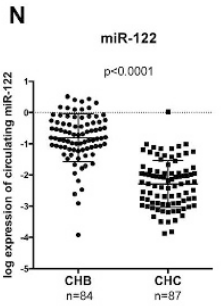

O
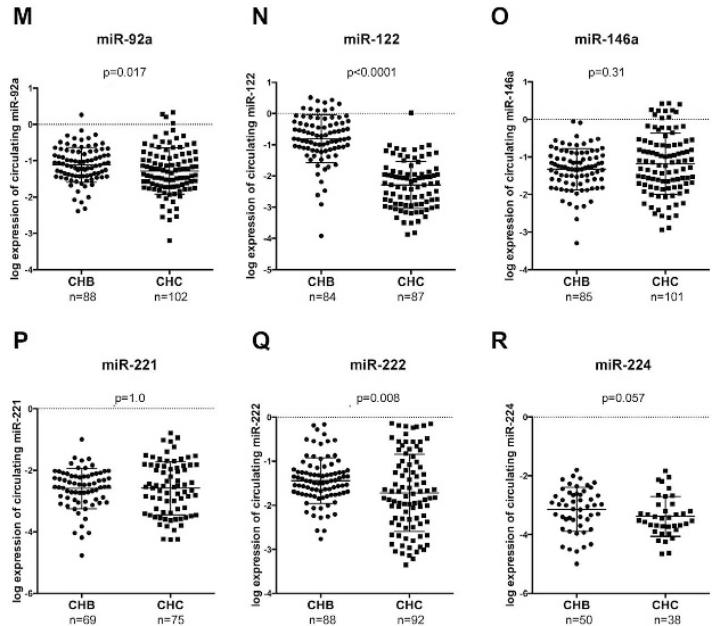

Figure 3. Comparison of the expression of serum and hepatic miRNAs in patients with chronic hepatitis B and C. The expression of serum (A-I) and hepatic (J-R) expression of miR-20a, miR-21, miR-29a, miR-92a, miR-122, miR-146a, miR-221, miR-222 and miR-224 was assessed by RT-qPCR and compared in patients with CHC and CHB. (A-I) In serum samples, The $\Delta \mathrm{Ct}\left(\Delta \mathrm{Ct}=2^{\Delta \mathrm{Cp} \text {,sample }}\right)$ of each miRNA was calculated and normalized to the $\Delta \mathrm{Ct}$ value of the exogenous $C$. elegans-miR-39 (cel-miR-39) in each serum. The log expression of the ratio miRNA/cel-miR-39 is shown as dot plot, each dot represents one patient (mean and standard deviation). (J-R) In liver samples, the $\Delta \mathrm{Ct}\left(\Delta \mathrm{Ct}=2^{\Delta \mathrm{Cp} \text {,sample }}\right)$ of each miRNA was calculated and normalized to the $\Delta \mathrm{Ct}$ value of SNORD44 in each biopsy. The log expression of the ratio miRNA/SNORD44 is shown as dot plot, each dot represents one patient (mean and standard deviation). The log expression of the ratio miRNA/SNORD44 is shown as dot plot, each dot represents one patient (mean and standard deviation). The Wilcoxon rank-sum test was used to compare miRNAs expression. 


\begin{tabular}{|l|c|c|c|c|c|c|c|c|}
\hline \multirow{2}{*}{} & \multicolumn{4}{|c|}{ Chronic hepatitis B } & \multicolumn{3}{c|}{ Chronic hepatitis C } \\
\cline { 2 - 10 } & \multicolumn{2}{|c|}{ ALT } & \multicolumn{2}{c|}{ AST } & \multicolumn{2}{c|}{ ALT } & \multicolumn{2}{c|}{ AST } \\
\cline { 2 - 10 } & $\mathbf{R}^{2}$ & p-value & $\mathbf{R}^{2}$ & p-value & $\mathbf{R}^{2}$ & p-value & $\mathbf{R}^{2}$ & p-value \\
\hline miR-20a & 0.17 & 0.11 & 0.13 & 0.24 & -0.08 & 0.45 & -0.04 & 0.68 \\
\hline miR-21 & $\mathbf{0 . 2 3}$ & $\mathbf{0 . 0 4}$ & $\mathbf{0 . 2 3}$ & $\mathbf{0 . 0 4}$ & 0.07 & 0.51 & 0.15 & 0.14 \\
\hline miR-27a & 0.08 & 0.56 & 0.09 & 0.51 & & & & \\
\hline miR-27b & 0.24 & 0.05 & 0.19 & 0.14 & & & & \\
\hline miR-29a & 0.16 & 0.15 & 0.11 & 0.31 & 0.10 & 0.39 & 0.13 & 0.27 \\
\hline miR-29c & 0.14 & 0.23 & 0.11 & 0.33 & & & & \\
\hline miR-92a & 0.08 & 0.49 & -0.02 & 0.86 & -0.05 & 0.66 & -0.05 & 0.61 \\
\hline miR-122 & $\mathbf{0 . 2 8}$ & $\mathbf{0 . 0 1}$ & 0.18 & 0.10 & $\mathbf{0 . 2 8}$ & $\mathbf{0 . 0 1}$ & 0.31 & 0.009 \\
\hline miR-146a & 0.01 & 0.92 & 0.07 & 0.55 & 0.03 & 0.80 & 0.00 & 0.99 \\
\hline miR-155 & -0.01 & 0.95 & 0.07 & 0.57 & & & & \\
\hline miR-221 & 0.18 & 0.15 & 0.18 & 0.14 & 0.00 & 0.98 & 0.03 & 0.82 \\
\hline miR-222 & 0.16 & 0.14 & 0.15 & 0.16 & 0.02 & 0.87 & 0.03 & 0.75 \\
\hline miR-224 & 0.05 & 0.75 & -0.03 & 0.84 & 0.13 & 0.44 & 0.10 & 0.55 \\
\hline
\end{tabular}

Table 5. Correlations between the expression of serum microRNAs and ALT and AST in CHB and CHC patients.

Several studies described additional analyses such as image analysis and liver gene expression assessment that may help to diagnose more precisely the stages of fibrosis from liver biopsy ${ }^{39-41}$. Moreover, the identification of genes and miRNAs which expression is modified at different stages of fibrosis is relevant to elucidate its molecular mechanisms.

Whereas the progression of fibrosis is a common feature of both $\mathrm{CHB}$ and $\mathrm{CHC}$, it is likely that the molecular mechanisms of fibrosis may be specific to each type of virus. Indeed, some miRNAs were differentially expressed the same way in F3-F4 vs F1-F2 patients with CHC and $\mathrm{CHB}$ and some miRNAs showed strong variations in between patients with $\mathrm{CHB}$ and in those with $\mathrm{CHC}$ independently of the stage of fibrosis (Fig. 3).

Our results suggest that the reduction of hepatic miR-122 and the increase of hepatic miR-224 may be a common feature of $\mathrm{CHB}$ and $\mathrm{CHC}$-induced fibrosis (Figs 1 and 2). We, and others, already reported the reduction of hepatic miR-122 in patients with $\mathrm{CHC}$, at later stages of fibrosis ${ }^{20,42}$. It might be suggested that the pool of functional hepatocytes decreases at the most advanced stages of fibrosis (F3-F4). Since the expression of hepatic miR-122 is assessed from biopsy of the whole liver, the reduction of miR-122 expression, we observed, may be a consequence of the reduction of the number of hepatocytes. Indeed, miR-122 is mainly expressed in hepatocytes whereas SNORD44 which is used for normalization is expressed at the same level in the different types of hepatic cells. Another explanation for the reduction of hepatic miR-122 in patients with F3-F4 may be the activation of HSCs and viral infection that were both associated with a decrease of miR-122 (reviewed in ref. 25).

Upregulation of hepatic miR-224 has been described in various types of cancer including HCC ${ }^{43,44}$. Thereby, the increased hepatic expression of miR-224 in patients with F3-F4 (Fig. 1) might emphasize that those patients are more likely to develop HCC compared to patients with F1-F2 who show lower level of hepatic miR-224. Interestingly, a previous report suggested that serum miR-224 was a reliable biomarker for the detection of early-stage of $\mathrm{HCC}^{45}$. This common characteristic observed in both $\mathrm{CHB}$ and $\mathrm{CHC}$ patients might represent interesting targets for anti-fibrotic therapies.

Hepatic miR-146a was increased in CHC patients compared to $\mathrm{CHB}$ patients. Recent data reported that miR-146a was promoting viral replication and deregulating metabolic pathways associated with liver disease ${ }^{46}$. The deregulation of miR-146a leading to the pathogenesis of liver disease may be specific of HCV infection. Interestingly, hepatic miR-122 was increased in CHC compared to CHB patients (Fig. 3). Whereas miR-122 stimulates HCV replication, miR-122 was suggested to inhibit the replication of $\mathrm{HBV}^{21}$. Therefore, in $\mathrm{CHC}$ hepatic miR-122 may be maintained to facilitate viral replication whereas in CHB miR-122 may be decreased to limit the inhibition of viral replication.

Serum miR-122 and miR-21 were weakly correlated with ALT and AST in CHB and CHC patients (Table 5). Moreover hepatic miR-122 was inversely correlated with AST and ALT. The current assumption regarding serum abundance of miRNAs is that miRNAs are released in the blood stream via an active and a passive release ${ }^{47}$. Thus, miR-21 and miR-122 may be passively released in the bloodstream following the lysis of hepatocytes. Interestingly, in CHB patients, while hepatic miR-122 was reduced compared to patients with CHC, serum miR-122 was 28 times higher than in CHC patients (Fig. $2 \mathrm{~N}$ ). These results illustrate the balance in between hepatic and serum miR-122. Since miR-122 is almost uniquely expressed in the liver, its presence in the serum is most probably due to a modification of its expression within hepatocytes. Serum miR-20a, miR-29a, miR-92a, miR-146a, miR-221, miR222 and miR-224 were not correlated with AST or ALT suggesting that these miRNAs may be selectively released. However, besides miR-122 the miRNAs assessed in our study are expressed by multiple types of cells therefore their presence in the serum may be a consequence of a deregulation in other tissues. Interestingly, hepatic miR20a and miR-92a were inversely correlated to the levels of ALT and AST (Table S9) whereas serum miR-20a and miR-92a were not correlated with AST and ALT (Table 5). These results support the hypothesis that the release of some miRNAs including miR-20a and miR-92a may be a consequence of the activation of different type of cells. 
The expression of miR-21 in increased in pulmonary ${ }^{48}$ and renal fibrosis ${ }^{49}$. However, we found no up-regulation of hepatic miR-21 in CHB and CHC suggesting that miR-21 may not be involved in liver fibrosis.

When, we started this project, we made the decision to develop a selective approach rather than a global one for the following reasons: (i) because we support a physiopathology approach, (ii) because of the rarity of our serum and liver samples and finally (iii) because of cost issues. We do agree that this choice implies a limited number of miRNAs studied and that a global approach would have increased the number of hits. However, using this approach we developed 2 models to predict severe fibrosis and cirrhosis that remain both robust and relevant as compared to other tests such as APRI and FIB-4.

Our study supports the relevance to develop 2 models for the diagnosis of fibrosis specifically in patients with $\mathrm{CHB}$ and $\mathrm{CHC}$. The model for patients with $\mathrm{CHB}$ (combining serum miR-122, serum miR-222, platelet count and ALP), and the model for patients with CHC (combining hepatic miR-122, hepatic miR-224, platelet count, ALT and albumin) were able to discriminate patients with F3-F4 from those with F1-F2. With better diagnostic performance than APRI and FIB-4. Interestingly, we report a differential profile of expression of miRNAs according to the stage of fibrosis and the type of viral infection. These findings may help to elucidate the molecular mechanisms of CHB and CHC-induced liver fibrosis.

\section{Methods}

Patients and Ethics statement. Among the 305 patients consecutively included, 103 and 178 had respectively CHB and CHC (Table S2). Patients were eligible if they had an established diagnosis of chronic viral hepatitis B or C (detectable HCV-RNA and HBV-DNA for more than 6 months) and had fibrosis assessment by liver biopsy. Patients were excluded if they had evidence of co-infection or any other cause of chronic liver disease. No patients were receiving treatment at the time of serum or liver biopsy. No patients have HCC. This study conformed to the ethical guidelines of the 1975 Declaration of Helsinki, All participants gave their informed written consent for the use of biological samples and clinical records. All experimental protocols were approved by the Comité de protection des personnes (CPP) and the Commission Nationale de l'Informatique et des Libertés (CNIL).

Among the $\mathrm{CHB}$ cohort, 87 serum and 83 liver samples were available for the analysis. Among the $\mathrm{CHC}$ cohort, 105 serum and 93 liver samples were available (Table S10). Interestingly, serum samples were coupled with liver samples for 69 and 20 patients of the $\mathrm{CHB}$ and $\mathrm{CHC}$ cohorts, respectively (Table S10). Previous non-responders to IFN, PEG-IFN plus ribavirin or boceprevir/telaprevir plus PEG-IFN plus ribavirin were included in the group of patients with CHC. 45 among 105 serums and 22 among 93 biopsies were from previous non-responders.

Patients with $\mathrm{CHB}$ were all naïve of treatment at the time of both the liver biopsy and the serum.

Histopathological assessment of liver biopsies. Ultrasound guided percutaneous liver biopsies were performed. Liver biopsies were considered to be adequate if they were longer than $10 \mathrm{~mm}$ and/or had at least 12 portal tracts. Liver histology were all analyzed by an expert pathologist. The stage of fibrosis of liver biopsies was assessed according to the METAVIR score: F0. no fibrosis; F1, mild fibrosis (portal fibrosis without septa); F2, moderate fibrosis (portal fibrosis with rare septa); F3, advanced fibrosis (numerous septa without cirrhosis); F4, cirrhosis. Necroinflammation was assessed as follow: A0. no activity, A1, mild activity, A2, moderate activity, A3, severe activity ${ }^{50}$. Hepatic steatosis was graded according to the Brunt classification as: 0 (no hepatocytes affected by macrovesicular steatosis), 1 ( $0-33 \%$ hepatocytes affected), 2 (33-66\% involved), and 3 (>66\% hepatocytes affected $)^{51}$.

Non -invasive assessment of liver fibrosis. APRI and FIB-4 are scores derived from routine blood markers with are calculated as follow: FIB- $4=($ age $($ year $) \times$ AST $(\mathrm{IU} / \mathrm{L})) /\left(\right.$ platelet count $\left(10^{9} / \mathrm{L}\right) \times \mathrm{ALT}^{1 / 2}$ $(\mathrm{IU} / \mathrm{L}))^{52}, \mathrm{APRI}=\left(\mathrm{AST}(/ \mathrm{ULN}) /\right.$ platelet count $\left.\left(10^{9} / \mathrm{L}\right)\right) \times 100^{11}$.

A FIB-4 index less than 1.45 (Fib-4<1.45) predicts a Metavir score between F0 and F2 while a FIB-4 index equal to or above 3.25 (FIB- $4 \geq 3.25$ ) predicts F3 and F4. In patients with a FIB-4 index ranging from 1.45 to 3.25 $(1.45<$ FIB- $4<3.25)$, the stage of fibrosis cannot be determined and predicted accurately.

An APRI score less than or equal to 0.5 (APRI $\leq 0.5)$ predicts a Metavir score between F0 and F2. An APRI score equal to or above $1.5(\mathrm{APRI} \geq 1.5$ ) predicts a Metavir score of F3 or F4. In patients with an APRI score ranging from 0.5 to $1.5(0.5 \leq \mathrm{APRI} \leq 1.5)$, the stage of fibrosis cannot be determined and predicted accurately.

Rationale of miRNAs selection. To select the miRNAs candidates, we conducted a selective research using pubmed. We use the following keywords: miRNAs, liver fibrosis, HCV and HBV either combined by two (miRNAs and liver fibrosis, miRNAs and HCV or miRNAS and HBV) or by three (miRNAs and liver fibrosis and HCV or miRNAs and liver fibrosis and HBV). The 13 miRNAs selected were either at least cited once in a major journal, or several times in minor journals. The 13 miRNAs selected were associated with: (1) the HCV and HBV life cycle and/or (2) the liver, cardiac and renal fibrosis and/or (3) the regulation of HSC activation and/or (4) HCC (Table S11).

MiR-20a and miR-92a are upregulated in serums of CHC patients compared to both healthy patients and those with fibrosis unrelated to HCV infection ${ }^{53}$. Serum and hepatic miR-21 is upregulated in patients with cirrhosis compared to healthy patient ${ }^{54}$. MiR-29 targets mRNAs of genes coding for extracellular matrix coding proteins and inhibits the proliferation of hepatic stellate cells (HSC). MiR-29 is inhibited by HCV and is down-regulated during fibrosis ${ }^{55}$. MiR-146a inhibits activation and proliferation of HSC and is down-regulated during fibrosis in mice models ${ }^{56}$. Increased expression of miR-221 and miR-222 indicates the activation of HSC and the progression of liver fibrosis ${ }^{27}$. Increased expression of miR-224 was associated with the development of HCC ${ }^{43,44}$ (Table S11). 
Chronic hepatitis B and C are main causes of hepatocellular carcinoma. Deregulation of several miRNAs has been associated with hepatocellular carcinoma ${ }^{57-59}$. Therefore, we investigated the potential of miRNAs related to hepatocellular carcinoma to be associated with the different stages of fibrosis. We assessed the expression of miR26a/-26b, miR-199a/b-3p and miR-223 in serum and liver samples of patients with CHB or CHC (Supplementary Figures 5 and 6 and Tables S12 and S13). None of these miRNAs was significantly deregulated in patients with F3-F4 compared to those with F0-F1-F2 (Supplementary Figures 5 and 6).

Total RNAs extraction from biopsies and serums. From liver tissues. Frozen liver biopsy specimens were crushed and dissolved in $1 \mathrm{ml}$ of iced RNAble (Eurobio). Chloroform was added; the upper phase was collected after centrifugation and added to the same volume of isopropanol. RNA was precipitated, and washed in $80 \% \mathrm{Et}-\mathrm{OH}$. The pellet was dissolved in RNase and DNase free $\mathrm{H}_{2} \mathrm{O}^{60}$. Optical density of total RNA was measured at $260 \mathrm{~nm}$.

From serums. Total RNAs were extracted from $400 \mu \mathrm{l}$ of serum (MiRvana, Life science). Since there is no reliable serum miRNA stably expressed in the serum, $5.6 \times 10^{8}$ copies of Caenorhabdditis elegans miR-39 (cel-miR-39, Qiagen) was added to each serum sample before the extraction ${ }^{20,60}$.

MiRNAs quantification. The miRNAs content in both liver and serum samples was analysed by Taqman RT-qPCR. Quantitative values are obtained from the crossing point (Cp), number at which the increase in the signal associated with exponential growth of PCR products begins to be detected using Light Cycler ${ }^{\circledR} 480$ System Software (Roche Diagnostics), according to the manufacturer's manuals. Briefly, $1 \mathrm{ng}$ of the reverse transcription product was used to assess the expression of each miARNs. The expression of hepatic microRNAs was normalized to the amount of a control small RNA, SNORD $44^{20,60}$ whereas the amount of serum microRNAs was normalized to the amount of the spiked-in cel-miR-39.

All the results were expressed as the $\mathrm{N}$-fold differences in target gene expression relative to SNORD44 and cel-miR-39, and termed Ntarget. Ntarget was determined as Ntarget $=2 \Delta$ Cpsample. The $\Delta$ Cp value was determined by subtracting the average Cp value of the target miRNA from the average Cp value of SNORD44 (liver biopsy samples) and cel-miR-39 (serum samples).

Statistical analysis. Data are presented as medians and interquartile range for continuous variables and frequencies with percentages for qualitative variables. Marginal association between single variables and F3-F4 fibrosis was assessed by a Wilcoxon rank-sum test for quantitative variables and Fisher's exact test. Multiple logistic regressions were used to determine a set of variables independently associated with F3-F4 fibrosis. Variables associated with intervention at a 0.05 level were considered in the multiple models. The model selections were performed using a backward stepwise variable algorithm and Akaike Criteria. Odds Ratio (OR) are given, with their 95\% Confidence intervals (95\% CI). Performance of models and markers was assessed using Area under the Curve (AUC) and its 95\% CI. Cut-off values for the prediction of patients with F3-F4 fibrosis was determined by maximizing the Youden index. Sensitivity, Specificity were directly estimated with their 95\% CI. Predictive positive value (PPV) and Negative predictive value were estimated using a prevalence of F3-F4 fibrosis in the population of $20 \%$. All tests were 2 sided, and a value $\mathrm{P}<0.05$ was considered statistically significant. Statistical analyses were carried out with $\mathrm{R}$ (version 3.1.0).

\section{References}

1. Schweitzer, A., Horn, J., Mikolajczyk, R. T., Krause, G. \& Ott, J. J. Estimations of worldwide prevalence of chronic hepatitis B virus infection: a systematic review of data published between 1965 and 2013. Lancet 386, 1546-1555 (2015).

2. de Martel, C., Maucort-Boulch, D., Plummer, M. \& Franceschi, S. World-wide relative contribution of hepatitis B and C viruses in hepatocellular carcinoma. Hepatology 62, 1190-1200 (2015).

3. European Association for Study of, L. EASL. Recommendations on Treatment of Hepatitis C 2015. Journal of hepatology (2016).

4. European Association For The Study Of The, L. EASL. Clinical practice guidelines: Management of chronic hepatitis B virus infection. Journal of hepatology 57, 167-185 (2012).

5. Panel, A. I. H. G. Hepatitis C guidance: AASLD-IDSA recommendations for testing, managing, and treating adults infected with hepatitis C virus. Hepatology 62, 932-954 (2015).

6. Terrault, N. A. et al. AASLD guidelines for treatment of chronic hepatitis B. Hepatology 63, 261-283 (2016).

7. Regev, A. et al. Sampling error and intraobserver variation in liver biopsy in patients with chronic HCV infection. The American journal of gastroenterology $97,2614-2618$ (2002).

8. Degos, F. et al. Diagnostic accuracy of FibroScan and comparison to liver fibrosis biomarkers in chronic viral hepatitis: a multicenter prospective study (the FIBROSTIC study). Journal of hepatology 53, 1013-1021 (2010).

9. European Association for Study of, L. \& Asociacion Latinoamericana para el Estudio del, H. EASL-ALEH. Clinical Practice Guidelines: Non-invasive tests for evaluation of liver disease severity and prognosis. Journal of hepatology 63, 237-264 (2015).

10. Ray Kim, W. et al. Evaluation of APRI and FIB-4 scoring systems for non-invasive assessment of hepatic fibrosis in chronic hepatitis B patients. Journal of hepatology 64, 773-780 (2016).

11. Wai, C. T. et al. A simple noninvasive index can predict both significant fibrosis and cirrhosis in patients with chronic hepatitis C. Hepatology 38, 518-526 (2003).

12. Sterling, R. K. et al. Development of a simple noninvasive index to predict significant fibrosis in patients with $\mathrm{HIV} / \mathrm{HCV}$ coinfection. Hepatology 43, 1317-1325 (2006).

13. Teshale, E. et al. APRI and FIB-4 are good predictors of the stage of liver fibrosis in chronic hepatitis B: the Chronic Hepatitis Cohort Study (CHeCS). Journal of viral hepatitis 21, 917-920 (2014).

14. Xiao, G., Yang, J. \& Yan, L. Comparison of diagnostic accuracy of aspartate aminotransferase to platelet ratio index and fibrosis-4 index for detecting liver fibrosis in adult patients with chronic hepatitis B virus infection: a systemic review and meta-analysis. Hepatology 61, 292-302 (2015).

15. in Guidelines for the Screening, Care and Treatment of Persons with Hepatitis C Infection (Geneva; 2014).

16. in Guidelines for the Prevention, Care and Treatment of Persons with Chronic Hepatitis B Infection (Geneva; 2015).

17. Szabo, G. \& Bala, S. MicroRNAs in liver disease. Nature reviews. Gastroenterology \& hepatology 10, 542-552 (2013). 
18. Jin, B. X. et al. MicroRNA panels as disease biomarkers distinguishing hepatitis B virus infection caused hepatitis and liver cirrhosis. Scientific reports 5, 15026 (2015).

19. Jopling, C. L., Yi, M., Lancaster, A. M., Lemon, S. M. \& Sarnow, P. Modulation of hepatitis C virus RNA abundance by a liver-specific MicroRNA. Science 309, 1577-1581 (2005).

20. Estrabaud, E. et al. Reduction of microRNA 122 expression in IFNL3 CT/TT carriers and during progression of fibrosis in patients with chronic hepatitis C. Journal of virology 88, 6394-6402 (2014).

21. Chen, Y. et al. A liver-specific microRNA binds to a highly conserved RNA sequence of hepatitis B virus and negatively regulates viral gene expression and replication. FASEB journal: official publication of the Federation of American Societies for Experimental Biology 25, 4511-4521 (2011).

22. Wang, S. et al. Loss of microRNA 122 expression in patients with hepatitis B enhances hepatitis B virus replication through cyclin G(1) -modulated P53 activity. Hepatology 55, 730-741 (2012).

23. Marcellin, P. \& Asselah, T. Long-term therapy for chronic hepatitis B: hepatitis B virus DNA suppression leading to cirrhosis reversal. Journal of gastroenterology and hepatology 28, 912-923 (2013).

24. Martinot-Peignoux, M. et al. Twelve weeks posttreatment follow-up is as relevant as 24 weeks to determine the sustained virologic response in patients with hepatitis $C$ virus receiving pegylated interferon and ribavirin. Hepatology 51, 1122-1126 (2010).

25. Bandiera, S., Pfeffer, S., Baumert, T. F. \& Zeisel, M. B. miR-122-a key factor and therapeutic target in liver disease. Journal of hepatology 62, 448-457 (2015).

26. Hsu, S. H. et al. Essential metabolic, anti-inflammatory, and anti-tumorigenic functions of miR-122 in liver. The Journal of clinical investigation 122, 2871-2883 (2012).

27. Ogawa, T. et al. MicroRNA-221/222 upregulation indicates the activation of stellate cells and the progression of liver fibrosis. Gut 61, $1600-1609$ (2012).

28. Jansen, F. et al. Endothelial microparticles reduce ICAM-1 expression in a microRNA-222-dependent mechanism. Journal of cellular and molecular medicine 19, 2202-2214 (2015).

29. Hellerbrand, Wang, S. C., Tsukamoto, H., Brenner, D. A. \& Rippe, R. A. Expression of intracellular adhesion molecule 1 by activated hepatic stellate cells. Hepatology 24, 670-676 (1996).

30. Li, Y., Chen, Y. \& Zhao, Y. The diagnostic value of the FIB-4 index for staging hepatitis B-related fibrosis: a meta-analysis. PloS one 9, e105728 (2014)

31. Mallet, V. et al. The accuracy of the FIB-4 index for the diagnosis of mild fibrosis in chronic hepatitis B. Alimentary pharmacology \& therapeutics 29, 409-415 (2009).

32. Kim, B. K. et al. Validation of FIB-4 and comparison with other simple noninvasive indices for predicting liver fibrosis and cirrhosis in hepatitis B virus-infected patients. Liver international: official journal of the International Association for the Study of the Liver 30, 546-553 (2010).

33. Fung, J. et al. Correlation of liver biochemistry with liver stiffness in chronic hepatitis B and development of a predictive model for liver fibrosis. Liver international: official journal of the International Association for the Study of the Liver 28, 1408-1416 (2008).

34. Seto, W. K. et al. A new model using routinely available clinical parameters to predict significant liver fibrosis in chronic hepatitis B. PloS one 6, e23077 (2011).

35. Gobel, T. et al. High prevalence of significant liver fibrosis and cirrhosis in chronic hepatitis B patients with normal ALT in central Europe. Journal of medical virology 83, 968-973 (2011).

36. Lemoine, M. et al. The gamma-glutamyl transpeptidase to platelet ratio (GPR) predicts significant liver fibrosis and cirrhosis in patients with chronic HBV infection in West Africa. Gut 65, 1369-1376 (2016).

37. Bedossa, P. \& Carrat, F. Liver biopsy: the best, not the gold standard. Journal of hepatology 50, 1-3 (2009).

38. Mehta, S. H., Lau, B., Afdhal, N. H. \& Thomas, D. L. Exceeding the limits of liver histology markers. Journal of hepatology 50, 36-41 (2009).

39. Calvaruso, V. et al. Computer-assisted image analysis of liver collagen: relationship to Ishak scoring and hepatic venous pressure gradient. Hepatology 49, 1236-1244 (2009).

40. Zhang, D. Y. et al. A hepatic stellate cell gene expression signature associated with outcomes in hepatitis C cirrhosis and hepatocellular carcinoma after curative resection. Gut 65, 1754-1764 (2016).

41. Asselah, T. et al. Liver gene expression signature of mild fibrosis in patients with chronic hepatitis C. Gastroenterology 129, 2064-2075 (2005).

42. Trebicka, J. et al. Hepatic and serum levels of miR-122 after chronic HCV-induced fibrosis. Journal of hepatology 58, 234-239 (2013).

43. Scisciani, C. et al. Transcriptional regulation of miR-224 upregulated in human HCCs by NFkappaB inflammatory pathways. Journal of hepatology 56, 855-861 (2012).

44. Gao, P. et al. Deregulation of microRNA expression occurs early and accumulates in early stages of HBV-associated multistep hepatocarcinogenesis. Journal of hepatology 54, 1177-1184 (2011).

45. Lin, L., Lu, B., Yu, J., Liu, W. \& Zhou, A. Serum miR-224 as a biomarker for detection of hepatocellular carcinoma at early stage. Clinics and research in hepatology and gastroenterology 40,397-404 (2016).

46. Bandiera, S. et al. HCV-induced up-regulation of miR-146a-5p in hepatocytes promotes viral infection and deregulates metabolic pathways associated with liver disease pathogenesis. Journal of virology 90, 6387-6400 (2016).

47. Roderburg, C. \& Luedde, T. Circulating microRNAs as markers of liver inflammation, fibrosis and cancer. Journal of hepatology 61, 1434-1437 (2014).

48. Liu, G. et al. miR-21 mediates fibrogenic activation of pulmonary fibroblasts and lung fibrosis. The Journal of experimental medicine 207, 1589-1597 (2010).

49. Chau, B. N. et al. MicroRNA-21 promotes fibrosis of the kidney by silencing metabolic pathways. Science translational medicine 4, 121ra118 (2012).

50. Bedossa, P. \& Poynard, T. An algorithm for the grading of activity in chronic hepatitis C. The METAVIR Cooperative Study Group. Hepatology 24, 289-293 (1996).

51. Brunt, E. M., Janney, C. G., Di Bisceglie, A. M., Neuschwander-Tetri, B. A. \& Bacon, B. R. Nonalcoholic steatohepatitis: a proposal for grading and staging the histological lesions. The American journal of gastroenterology 94, 2467-2474 (1999).

52. Vallet-Pichard, A., Mallet, V. \& Pol, S. FIB-4: a simple, inexpensive and accurate marker of fibrosis in HCV-infected patients. Hepatology 44, 769, author reply 769-770 (2006).

53. Shrivastava, S. et al. Up-regulation of circulating miR-20a is correlated with hepatitis $C$ virus-mediated liver disease progression. Hepatology 58, 863-871 (2013).

54. Zhao, J. et al. MiR-21 simultaneously regulates ERK1 signaling in HSC activation and hepatocyte EMT in hepatic fibrosis. PloS one 9, e108005 (2014).

55. Roderburg, C. et al. Micro-RNA profiling reveals a role for miR-29 in human and murine liver fibrosis. Hepatology 53, 209-218 (2011).

56. Du, J. et al. MiR-146a-5p suppresses activation and proliferation of hepatic stellate cells in nonalcoholic fibrosing steatohepatitis through directly targeting Wnt1 and Wnt5a. Scientific reports 5, 16163 (2015).

57. Hou, J. et al. Identification of miRNomes in human liver and hepatocellular carcinoma reveals miR-199a/b-3p as therapeutic target for hepatocellular carcinoma. Cancer cell 19, 232-243 (2011). 
58. Ji, J. et al. MicroRNA expression, survival, and response to interferon in liver cancer. The New England journal of medicine 361, $1437-1447$ (2009).

59. Wong, Q. W. et al. MicroRNA-223 is commonly repressed in hepatocellular carcinoma and potentiates expression of Stathmin1. Gastroenterology 135, 257-269 (2008).

60. Estrabaud, E. et al. IFI35, mir-99a and HCV genotype to predict sustained virological response to pegylated-interferon plus ribavirin in chronic hepatitis C. PloS one 10, e0121395 (2015).

\section{Acknowledgements}

This work was supported by the French National Agency for Research on AIDS and Viral Hepatitis (ANRS) (SF/ $\mathrm{CB} / \mathrm{N}^{\circ} 1178$ ) and the ANR through ERA-NET Infect-ERA program (ANR-13-IFEC-0002-01). KA was supported by a grant from ANRS (NM/nº24/CSS 7/AO 2012-1).

\section{Author Contributions}

K.A. performed most of the experiments. K.A., E.E. and T.A. wrote the paper. M.R.-R. performed the statistical analysis. P.B. performed analysis and staging of liver biopsy specimens. E.E. and T.A. conceived and designed the study, and supervised all aspects of the work. T.A., N.B., M.M.-P. and M.L. provided liver and serum samples. K.A., S.D., X.T., C.-H.G., E.P., M.V., P.M., I.B., E.E. and T.A. discussed the results and implications, and commented on the paper.

\section{Additional Information}

Supplementary information accompanies this paper at http://www.nature.com/srep

Competing financial interests: The authors declare no competing financial interests.

How to cite this article: Appourchaux, K. et al. MicroRNA-based diagnostic tools for advanced fibrosis and cirrhosis in patients with chronic hepatitis B and C. Sci. Rep. 6, 34935; doi: 10.1038/srep34935 (2016).

(c) (i) This work is licensed under a Creative Commons Attribution 4.0 International License. The images or other third party material in this article are included in the article's Creative Commons license, unless indicated otherwise in the credit line; if the material is not included under the Creative Commons license, users will need to obtain permission from the license holder to reproduce the material. To view a copy of this license, visit http://creativecommons.org/licenses/by/4.0/

(c) The Author(s) 2016 